\title{
ASSESSING THE SUITABILITY OF SIMULATED SAR SIGNATURES OF DEBRIS FOR THE USAGE IN DAMAGE DETECTION
}

\author{
S. Kuny ${ }^{\mathrm{a}, \mathrm{b}}$, H. Hammer ${ }^{\mathrm{a}}$, K. Schulz ${ }^{\mathrm{a}}$ \\ ${ }^{a}$ Fraunhofer IOSB, Institute of Optronics, System Technologies and Image Exploitation, Ettlingen, Germany - (silvia.kuny, \\ horst.hammer, karsten.schulz)@iosb.fraunhofer.de \\ ${ }^{\mathrm{b}}$ Institute of Photogrammetry and Remote Sensing IPF, Karlsruhe Institute of Technology KIT, Germany
}

Commission III, ICWG III/VII

KEY WORDS: SAR simulation, debris, damage detection, texture features

\begin{abstract}
:
When disasters strike in urban areas, the most important issue is to direct rescue forces to the most heavily destroyed areas. SAR images, because of their independence from daylight and weather conditions, are the remote sensing tool of choice in these cases. However, often no pre-event image is available, so change detection cannot be performed. Thus, we aim at extracting areas of debris from a single post-event SAR image using textural features. We want to be independent of real samples of debris sites by using simulated SAR image chips. Previous work has shown that in this way we detect all major sites of debris, e.g. caused by collapsed buildings. However, the screening process also detects many other areas, especially high vegetation and gravel. In order to rule these areas out from the analysis, it is important to also simulate these classes of objects. The simulated chips can then be used in a classifier, specifically a random forest, to rule out these causes of false alarms.
\end{abstract}

\section{INTRODUCTION}

Natural disasters, in particular earthquakes, cause a strong demand for a fast and reliable detection of structural damages. Due to the independence of weather and lighting conditions and the consequentially ensured image availability, many approaches are based on SAR imagery, occasionally in combination with ancillary data. However, the likely and rather challenging case of having neither pre-event image nor additional data available is treated rarely. In X-band SAR imagery the most prominent indication for structural damages is the signature caused by heaps of debris surrounding the buildings. Due to its coarse texture, debris can be separated rather well from other signatures caused by urban formations. However, there are several sources, most importantly high vegetation that feature a very similar texture and thus make the classification approach considerably more difficult. Previous work addressed the search for suitable textural features to describe these types of more or less random textures and the advantages of using simulated data as training samples for damage detection purposes. Essentially, this entails the prospect of creating generic samples, which are unaffected by random factors and independent from the actual SAR image that is to be investigated. Since basing the learning step of a classifier on simulated data requires the simulations to be accurate in respect to radiometry, a direct comparison between simulated and real data is a crucial step.

This paper aims at validating the usage of simulated SAR textures of debris in damage detection by demonstrating the good agreement of the simulations with TerraSAR-X Spotlight textures. For this, suitable texture features are used to visualize and assess the similarity. Furthermore, sources of similar texture, such as high vegetation and gravel, are addressed, hereby validating several aspects of simulating training data. The simulation of textures like gravel is managed solely by backscattering characteristics, due to its small-scale surface roughness, whereas textures like debris and vegetation additionally rely on the accuracy of the $3 \mathrm{~d}$ models. So the aim is to not only verify the choice of material backscattering properties that are used in the simulation process, but also the assumptions that are made for $3 \mathrm{~d}$ models, in particular the heaps of debris.

The paper is structured as follows: In Section 2 we introduce the simulation of generic texture samples and give examples of the $3 \mathrm{~d}$ models used in the simulation process. In Section 3 we compare the simulated to real signatures and assess the similarity using grey level histograms and textural features. Section 4 contains some results on the classification of real samples. We finish with the conclusions and an outlook to future plans in Section 5 .

\section{SIMULATION OF GENERIC TEXTURE SAMPLES}

With the main goal of detecting damaged and destroyed buildings, our focus is on defining and setting limits to the characteristics of the SAR texture caused by debris. By resorting to simulations as source for this texture information we have got essential advantages in the long run. For one, the simulation process provides a controlled environment, thus ruling out disruptive factors such as overlaying signatures. Furthermore, simulated signatures are bound to be more generic than real samples. For a training sample this is a good quality. And most notably, the simulation of texture information makes us independent from the actual SAR image to be investigated, not just regarding the signature of debris, but potential further types of SAR signatures. For this approach it is essential that the simulations are realistic and comparable to real signatures. First results show that a classifier trained with simulated samples can successfully separate vegetation from debris (Kuny, 2016). However, to validate the usage of simulations in this way we conduct a direct comparison of real and simulated textures. 


\subsection{Simulator}

For the generation of radiometrically correct SAR images we use CohRaS ${ }^{\circledR}$, a ray-tracing based SAR simulator developed at Fraunhofer IOSB. The main characteristics of $\mathrm{CohRaS}^{\circledR}$ are described in (Hammer, 2009). Even though CohRaS ${ }^{\circledR}$ does not simulate raw data, the main characteristics of backscattering and dihedral and trihedral reflections are dealt with in a consistent way, so that the images simulated using $\mathrm{CohRaS}^{\circledR}$ can be used in direct comparison with real images.

\subsection{Textures of interest}

As the most prominent signature of damaged and destroyed buildings, the texture of debris in all of its forms poses our main interest. Previous studies (Kuny, 2014) show that this signature indeed is distinguishable rather well from most signatures caused by urban structures. However, several types of areas cause a signature that is very similar to that of debris, most importantly vegetation and gravel. In order to demonstrate, that the texture simulations are not only feasible and realistic, but also accurate enough to be realistically distinguishable, those two texture types are also focus of this study.

Crucial for the simulation of a realistic SAR signature are, first of all, the parameter settings describing the radiometric backscattering characteristics of the varying materials. The scattering pattern of each material is specified using five parameters. Furthermore, the suitability of the $3 \mathrm{~d}$ model is decisive for a realistic texture simulation. Particularly, the macroscopic surface roughness has to be representative.

2.2.1 Debris: It was assumed that heaps of debris are made up mostly of stone bricks and wooden materials. We used generalized $3 \mathrm{~d}$ models of heaps of debris, consisting of a large amount of cuboids of different sizes and varying materials. In order to cover the wide range of possible debris characteristics a range of $3 \mathrm{~d}$ models with varying configurations and a different macroscopic surface roughness were used for the simulations. An exemplary $3 \mathrm{~d}$ model is shown in Figure 1.

2.2.2 High Vegetation: In contrast to sensors with larger wavelengths, $\mathrm{X}$-band microwave does not penetrate the canopy of a tree. Hence, almost all backscattering is caused by leaves. For the $3 \mathrm{~d}$ tree model in our study this means a high level of detail and a very large number of polygons. The sample tree depicted in Figure 2 consists of approximately 200,000 polygons, most of which are located in the leaves.

2.2.3 Gravel: Regarding the X-band wavelength of $3.1 \mathrm{~cm}$, gravel has a very small-scale surface roughness. This means, that with respect to the $3 \mathrm{~d}$ modelling it is not necessary to employ a realistic scenario, actually consisting of millions of small gravel stones. It is sufficient to capture the (large scale) statistical characteristics of the surface, which can be achieved using simply a flat plane, which is endowed with a material consistent with a very rough and non-specular surface. This is the approach we adopted in this study.

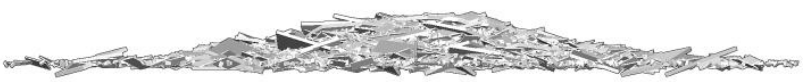

Figure 1. Exemplary 3d model for a heap of debris.

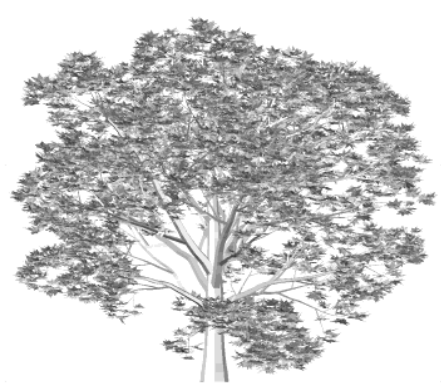

Figure 2. Sample tree model used to simulate the texture of high vegetation.

\section{COMPARISON TO REAL TEXTURE}

The real texture samples of debris, vegetation and gravel are taken from a TerraSAR-X image of Christchurch, New Zealand, recorded after the earthquake in 2011. All in all, 33 sets of debris samples were extracted for the study, as well as some gravel and vegetation samples.

\subsection{Gray value distribution}

The similarity between the SAR texture of debris and the textures of vegetation and gravel makes for rather similar gray value distributions. Figure 3 (left) shows the normalized amplitude histograms of the TerraSAR-X debris samples (orange) to the samples of vegetation (blue). Figure 3 (right) shows the comparison between debris and gravel.

As a first verification towards a correct simulation, the gray value distributions of the simulated texture samples show a very similar character, for the three texture types respectively. Corresponding histograms are shown in Figure 4. The similarity of the histograms of real and simulated data show that the adopted approach is feasible, i.e. on the one hand the used $3 \mathrm{~d}$ models and the chosen material properties are similar to those found in the real signatures, and on the other hand, that indeed the adopted approach of $\mathrm{CohRaS}^{\circledR}$ for direct simulation of the images without creating raw data is close enough to the real image formation process in this case.
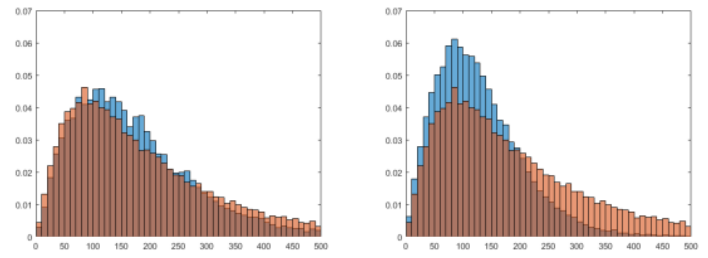

Figure 3. Gray value histogram of real debris amplitude samples (orange) in comparison to vegetation (left) and gravel (right).
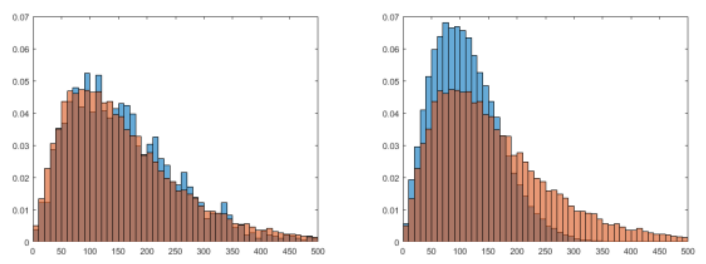

Figure 4. Gray value histogram of simulated debris amplitude samples (orange) in comparison to vegetation (left) and gravel (right). 


\subsection{Statistical texture features}

The detection of debris-like structures in a SAR image is performed in a two-step approach (see Kuny, 2016). First, we use several texture features to discriminate debris-like textures from other signatures. This first step can be seen as a rough screening. While this approach leads to many false alarms, caused in many cases by vegetation and gravel, it greatly reduces the workload by already excluding large sections of the image from further analysis, while at least all of the larger areas of debris in the image that are visible to the sensor are detected.

The second step of our approach consists in the separation of the detection into several classes. As means to describe the texture of debris and to separate it from other signatures that are similar to the texture of debris, we found some statistics of the first order as well as some Haralick features (Haralick, 1973) to perform the best. These have been described in (Kuny, 2015) in detail. For the sake of completeness we include the results shown in (Kuny, 2015) again: The best-performing texture features that we also used in this study to separate debris from vegetation and gravel consist of first order statistics (standard deviation, variance) and features computed on the gray-level cooccurrence matrix (contrast, variance, sum entropy) and are depicted in Figure 5.
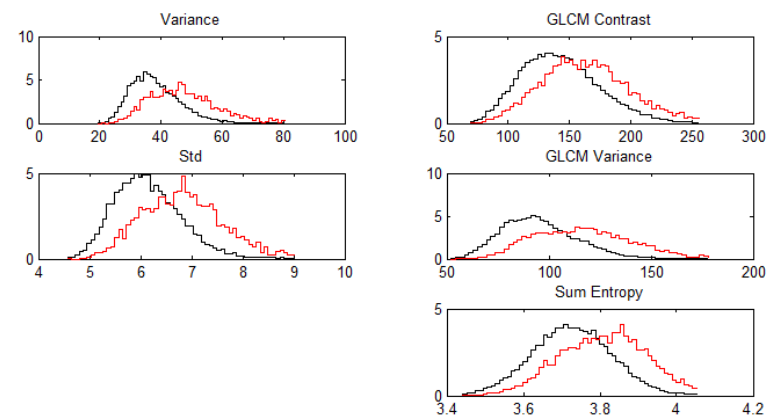

Figure 5. The best performing texture features regarding the discrimination of debris (red) and vegetation (black).

In order to have a visual impression of the feature results, a multidimensional scaling was applied. With Sammon projection (Sammon, 1969), a high-dimensional space is mapped to a lower dimensionality, whilst the structure of inter-point distances is tried to be preserved. To do so, the following error function is minimized:

$$
E=\frac{1}{\sum_{i<j} d_{i j}^{*}} \sum_{i<j} \frac{\left(d_{i j}^{*}-d_{i j}\right)^{2}}{d_{i j}^{*}}
$$

where $d_{i j}^{*}$ denotes the distance between the $\mathrm{i}^{\text {th }}$ and $\mathrm{j}^{\text {th }}$ objects in the original space and $d_{i j}$ is the distance between their projections.

In Figure 6 the feature clusters of debris (black), vegetation (green) and gravel (red) for the TerraSAR-X samples are depicted. It becomes clear that the signature of debris comprises a wide diversity of texture characteristics. This could be expected, since the variation of possible debris configurations and the involved materials has a much larger range than for example vegetation or gravel. The feature clusters of the three classes do overlap somewhat, which could be anticipated since all have a very similar signature. However, large parts of vegetation and especially of gravel seem to be clearly discriminable by these texture features.

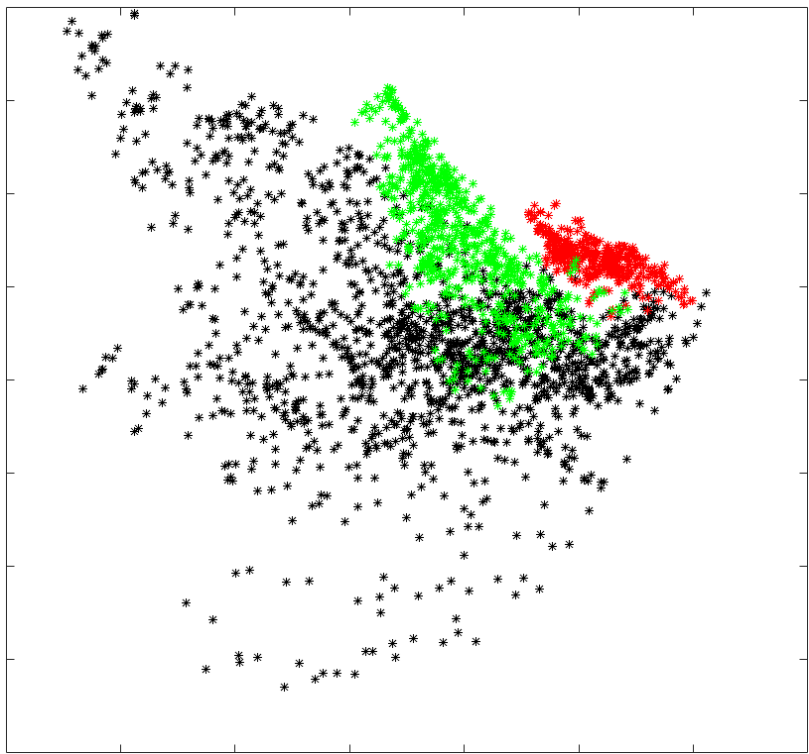

Figure 6. Mapped texture features regarding TerraSAR-X samples of debris (black), vegetation (green) and gravel (red).

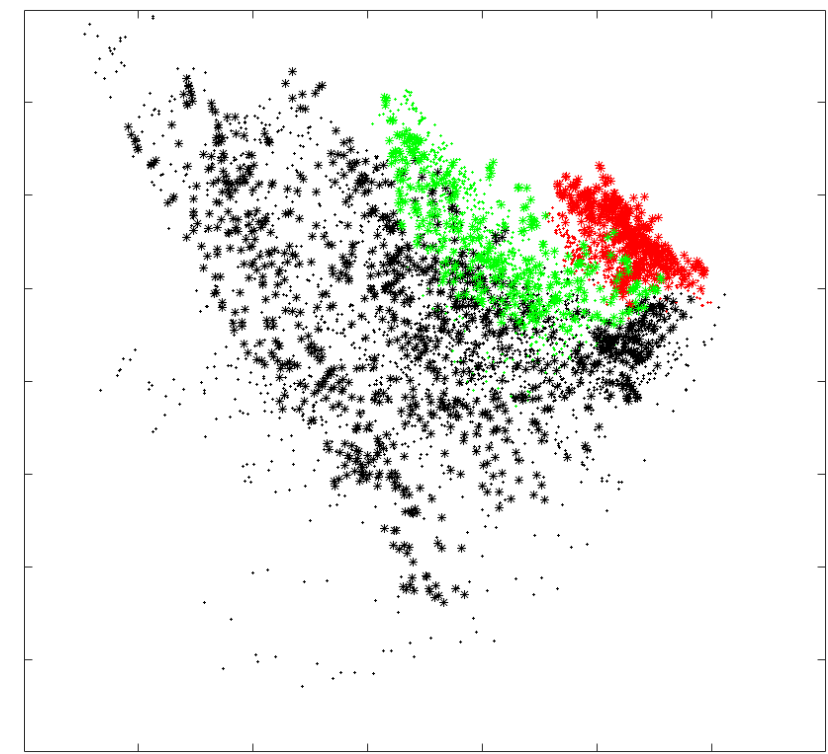

Figure 7. Mapped texture features regarding simulated samples of debris (black), vegetation (green) and gravel (red). For comparison, the clusters of Figure 3 are marked as well.

Accordingly, the texture features of the simulated samples of debris, vegetation and gravel were computed and a Sammon mapping was applied. In Figure 7 the resulting feature clusters are shown. To be comparable to Figure 6 a similar number of sample entities was chosen, whereas the feature clusters of the real samples are indicated by small-scale markers. All in all, the clustering shows a rather good agreement with features of real samples, confirming again the accuracy of the simulations of all three signature types. First attempts at separating vegetation from debris by using simulated samples already show good results. These results are described in an upcoming paper (Kuny, 2016). 


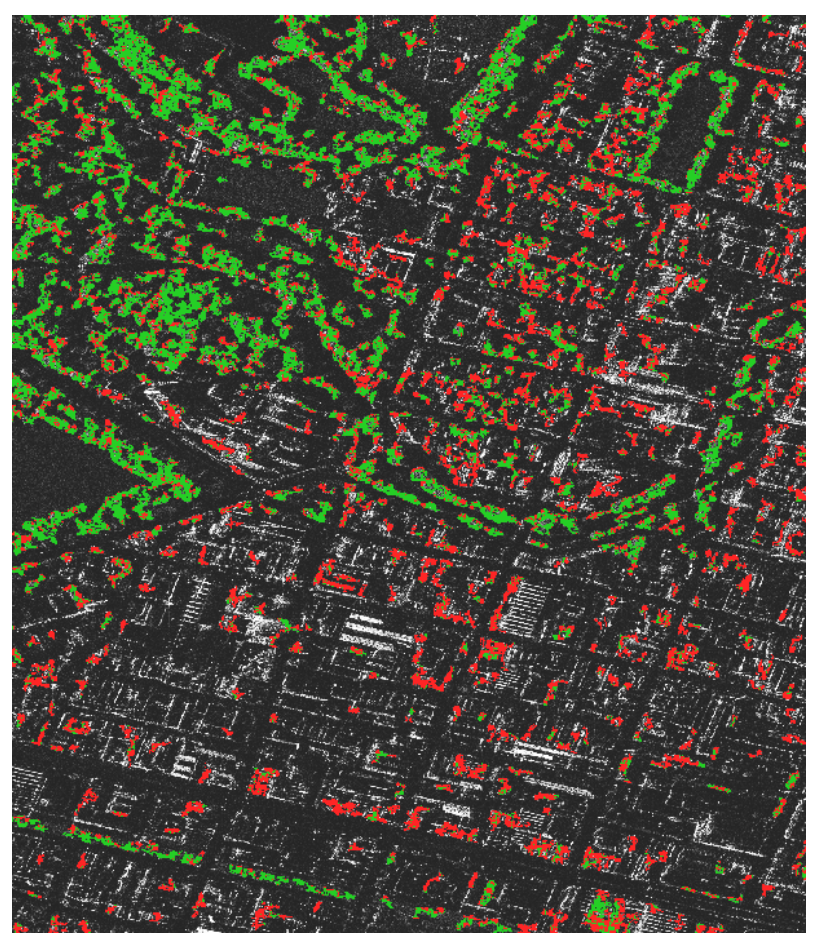

Figure 8. Subimage of the TerraSAR-X image of Christchurch. Separation of vegetation (green) from debris-like texture (red).

\section{CLASSIFICATION OF SAMPLES}

The classification of the real image samples into the three classes of debris, vegetation and gravel is performed using a random forest trained with the simulated signatures of the respective class. While this is still work in progress, first results of the classification have shown that the texture of vegetation can be separated very well from the debris textures using this approach (see also (Kuny, 2016)). Figure 8 shows a subimage of the Christchurch scene. The areas marked in red and green have been detected as being debris-like by a screening process using the full set of textural features. The areas marked in green have then been separated from the whole set of detections by the random forest classifier. A comparison with an available airborne optical image of very high resolution, recorded only few days after the earthquake, shows very good agreement with the detected vegetation areas (see Figure 9).

Figure 6 shows that the texture of gravel is rather more distinct from that of debris than the texture of vegetation, and is also quite distinct from the signature of vegetation. Thus, it is expected that gravel can be separated from both of the previous classes by the random forest classifier also, thus further reducing the number of false alarms while preserving all of the real areas of debris. However this will have to be verified.

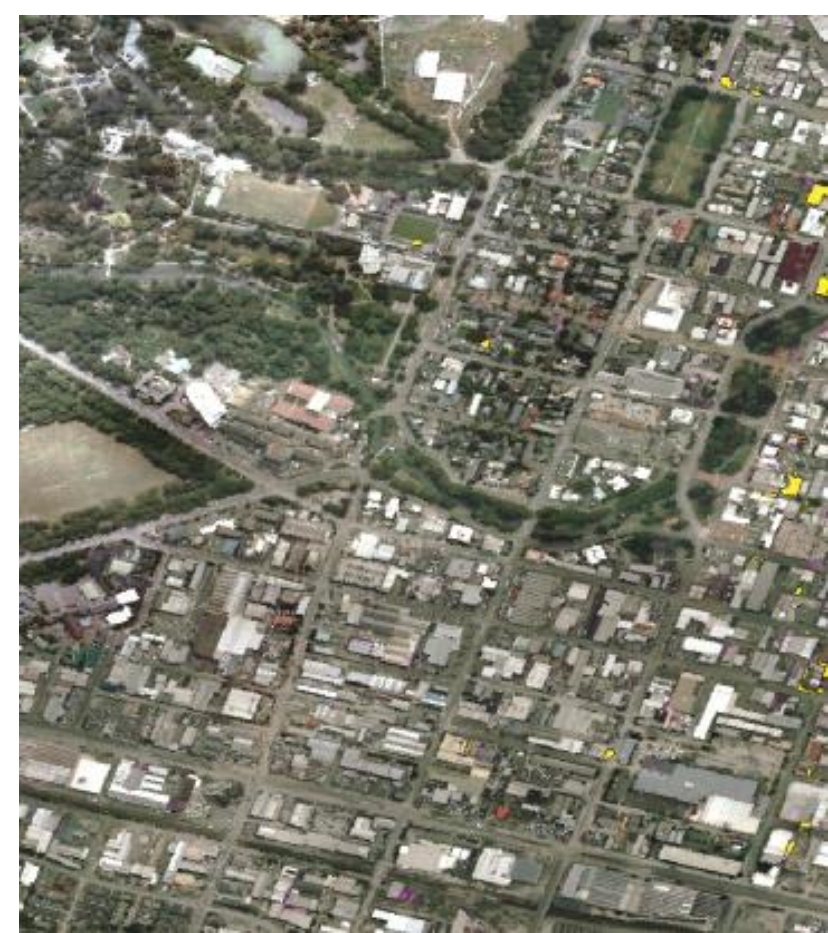

Figure 9. Subimage of the EO image of Christchurch corresponding to the SAR image in Figure 8. The EO image was provided by Land Information New Zealand.

Within the Christchurch scene, there are several areas of severely damaged buildings. These were extracted from the EO image manually. In Figure 10 we show two samples of such scenes in the TerraSAR-X image and the corresponding EO image. As can be seen from these details, the large heaps of debris caused by the collapse of the buildings have been found by the screener and have been correctly identified as being debris by the classifier.
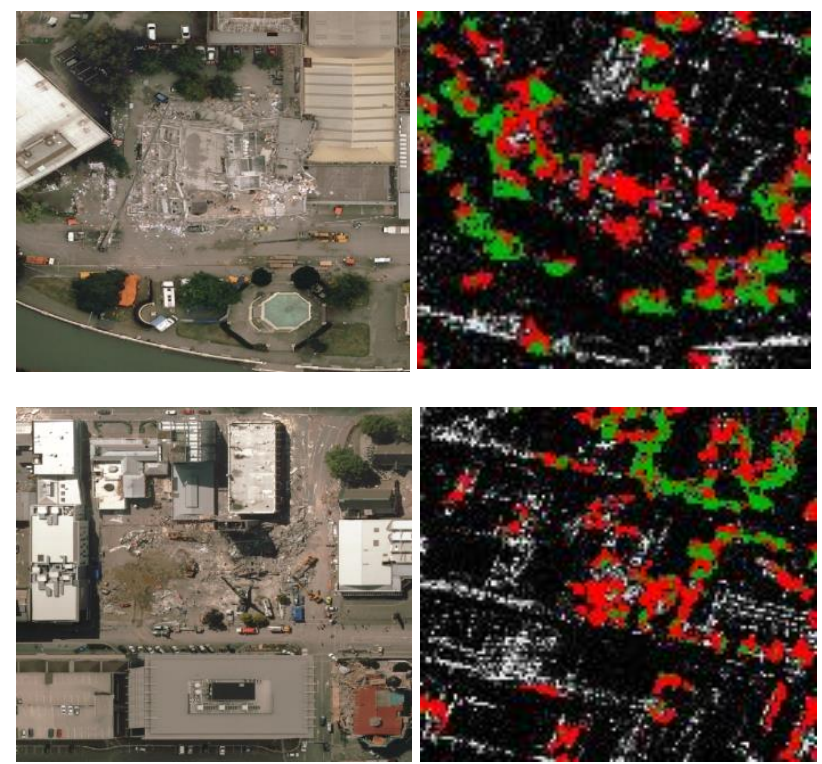

Figure 10. Two scenes of a destroyed building in the EO image (left) and their correct identification as debris(right). 


\section{CONCLUSIONS}

The use of simulated SAR textures of heaps of debris in damage detection has already shown promising results. In this paper an actual comparison of simulated and real textures of debris was conducted. Two sources of similar texture, namely vegetation and gravel, were simulated and included in the comparison. The good agreement of simulated textures of all three classes with those of real textures confirms several aspects of the simulation process, for one the choice of material backscattering properties that were used in the simulation process and secondly the assumptions that were made for the $3 \mathrm{~d}$ models.

The textural features used in this study were calculated for a wide variety of real and simulated samples of the three classes of debris, vegetation and gravel. The resulting points in feature space were projected to a $2 \mathrm{~d}$ plane using the Sammon projection. The resulting projected images show good agreement of the simulated and real areas in feature space. Furthermore, they show that the chosen features are able to separate the different classes. While some overlap areas were to be expected, given the similarity of the classes, they are separated enough to be used to discriminate the different classes with great certainty. This separability of the classes has already been demonstrated for debris and vegetation using a random forest classifier. We plan to expand the classification to a threeclass problem, discriminating between debris, gravel and vegetation. This can be seen as a major step to reduce the false alarms produced in the screening step of the two-step detection algorithm for the detection of areas of debris in urban areas after a disaster.

While the ruling-out of vegetation and gravel areas already greatly reduces the number of detected debris-like areas, there are other causes for misdetections. We plan to investigate these causes further and come up with more classes that can be described by generic simulations, as has been done for gravel and vegetation. In a second step, these new classes will be simulated and inserted into the classification. We expect, in this way, to be able to detect large areas of debris caused by natural disasters in urban areas more reliably without any pre-event data, thus enabling the guidance of rescue forces to the most damaged areas of a city, independent from daylight or weather conditions.

\section{REFERENCES}

Hammer, H., Schulz, K., 2009. Coherent Simulation of SAR Images, Proceedings of SPIE, Image and Signal Processing for Remote Sensing XV, doi: 10.1117/12.830380, Vol. 7477, pp. 74771G-1-9, 2009.

Haralick, R. M., Shanmugan, K., Dinstein, I., 1973. Textural features for image classification. IEEE Transactions on Systems, Man, and Cybernetics, Vol. 3, No. 6, pp. 610-621, 1973.

Kuny, S., Schulz, K., 2014, Debris detection in SAR imagery using statistics of simulated texture. $8^{\text {th }}$ Workshop on Pattern Recognition in Remote Sensing PRRS, 4 p., DOI 10.1109/PRRS.2014. 6914289, 2014.

Kuny, S., Hammer, H., Schulz, K., 2015. Discriminating between the SAR signatures of debris and high vegetation. Proceedings of IEEE Geoscience and Remote Sensing Symposium IGARSS, pp. 473-476, 2015.
Kuny, S., Hammer, H., Schulz, K., Hinz, S., 2016. Towards a Reliable Detection of Debris in High Resolution SAR Images of Urban Areas. Accepted for publishing in: Proceedings of European Conference on Synthetic Aperture Radar (EUSAR), 2016.

Sammon, J. W., 1969. A Nonlinear Mapping for Data Structure Analysis. IEEE Transactions on Computers, Volume 18 Issue 5, pp 401-409, 1969. 